Exploring the Design of Interactive Smart Textiles for Emotion Regulation Jiang, M., Bhömer, M., Liang, H.-N.

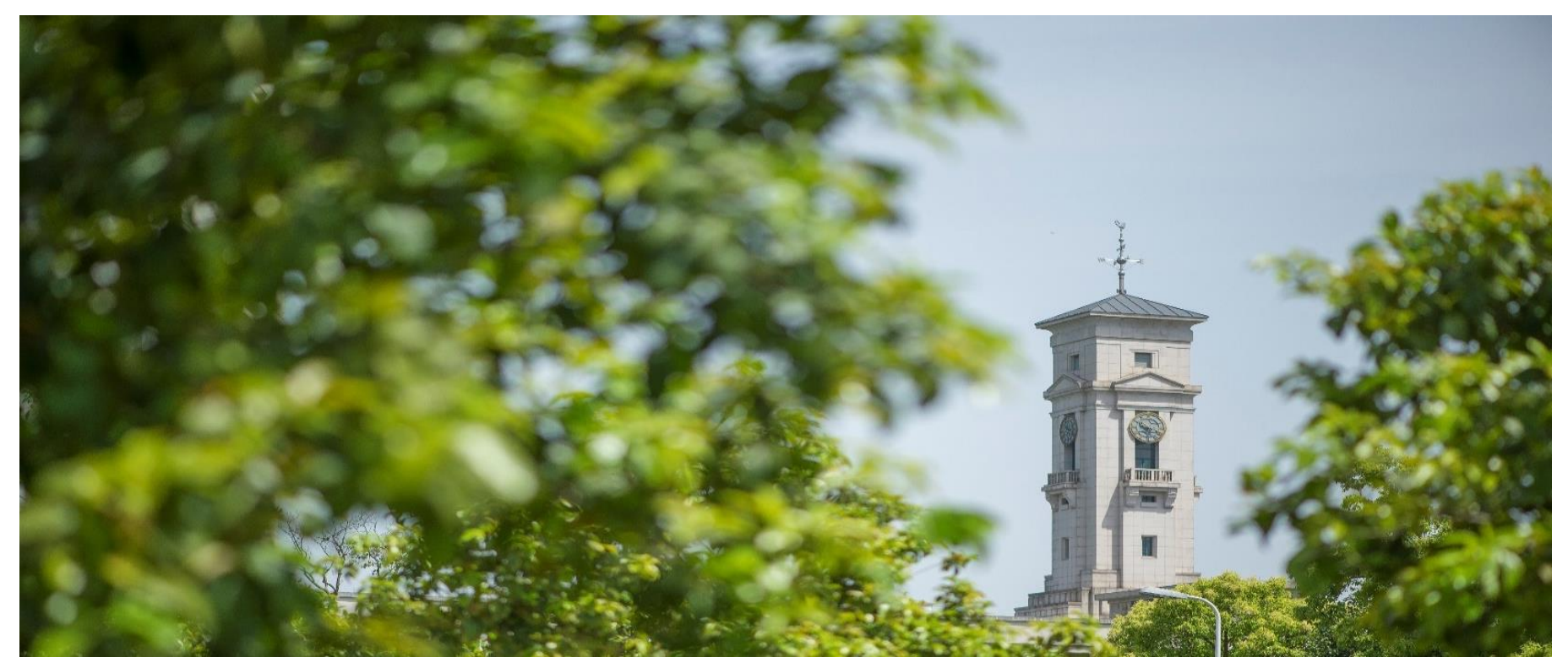


University of Nottingham Ningbo China, 199 Taikang East Road, Ningbo, 315100, China

First published 2020

This work is made available under the terms of the Creative Commons Attribution 4.0 International License:

http://creativecommons.org/licenses/by/4.0

The work is licenced to the University of Nottingham Ningbo China under the Global University Publication Licence:

https://www.nottingham.edu.cn/en/library/documents/researchsupport/global-university-publications-licence.pdf Nottingham 


\title{
Exploring the Design of Interactive Smart Textiles for Emotion Regulation
}

\author{
Mengqi Jiang ${ }^{1[0000-1111-2222-3333]}$, Martijn ten Bhömer ${ }^{21111-2222-3333-4444]}$ and Hai-Ning \\ Liang $^{3 *[2222-3333-4444-5555]}$ \\ ${ }^{1,3}$ Xi'an Jiaotong-Liverpool University, Ren'ai Road. 111, 215000 Suzhou, China \\ Mengqi.Jiang@xjtlu.edu.cn \\ HaiNing.Liang@xjtlu.edu.cn \\ ${ }^{2}$ University of Nottingham Ningbo China, Taikang East Road. 199, 315100 Ningbo, China \\ martijn.ten.bhomer@nottingham.edu.cn
}

\begin{abstract}
The present study aims to investigate the design of interactive textiles for emotion regulation. In this work we proposed a design which allows users to visualize their physiological data and help regulate their emotions. We used the Research through Design method to explore how physiological data could be represented in four different interactive textiles and how movement-based interaction could be designed to support users' understanding and regulation of their emotional state. After an initial user interview evaluation with several textile prototypes, light and vibration were selected as modalities within the biofeedbackbased interaction. A smart interactive shawl that reacts to changes in emotional arousal was designed to help the users know their emotion and adjust it, if necessary, with the support of electrodermal activity sensor and pressure-based sensors. The results of the second study showed that the smart shawl could help the user to visualize their emotions and reduce their stress level by interacting with it.
\end{abstract}

Keywords: Design, Smart Textiles, Emotion regulation, Interactive Textiles, Research though Design.

\section{Introduction}

Emotional fluctuations, caused by work stress, academic examinations, challenging relationships, physiological diseases, happen regularly in many people's lives and affect our behaviour and decision-making to a considerable extent. Despite emotions, such as depression, fear, anxiety, and nervousness being an essential part of the life experiences, it may not be ideal for people to be regularly trapped in an overwhelming emotional state for a long time without realizing it. We are encouraged to self-regulate our emotions, and it has been reported that emotion self-regulation plays a significant role in effectively restoring physical and mental health [30].

The prevalent strategies of emotion self-regulation include psychological counselling, self-reflection, physical exercise, yoga, meditation, and catharsis. Conventionally, 
people rely on their subjective will to regulate their emotions through different approaches; however, in extreme cases, maintaining a rational and calm mindset may not be possible, given that many people may occasionally be overwhelmed by strong emotions. Therefore, researchers have explored ways to support the process of emotion regulation with digital technology. Biofeedback, as a body-mind technique that involves using visual, auditory or haptic feedback to control involuntary body functions, can help the user better control body's reactions and behaviours and understand their internal emotional states more explicitly [35]. The classical biofeedback design is a kind of one-way augmented feedback; that is, users can reflect on their physiological state through augmented feedback. However, as Yu [34] mentioned, biofeedback can also be functional and allow bidirectional, dynamic, adaptive feedback base on user's states and interaction [33]. We hope that users have more autonomy in emotional regulation. In this research, we aim to help people self-regulate their emotions by not only providing biofeedback but also allowing the wearer to actively interact with the 'visualized' biofeedback on clothing that they typically wear. However, what remains unclear is how to design bidirectional adaptive biofeedback-based interaction to regulate emotion.

Although many HCI researches are paying attention to emotion engaged interaction design $[9,10,32]$, very few studies have explored how interactive design could be used to regulate emotion, especially with interactive textiles. Interactive textiles can sense the stimuli or signals from the user and the environment, then react and properly adapt to them [4]. Besides, interactive textiles have the advantage of traditional textiles, while also being able to integrate technology and can be unobtrusively worn close to the source of physiological signals - i.e., the user. As such, they are well suited for supporting users with needs of short- and long-term emotion self-regulation. The goal of this research is to explore how interactive textiles can be designed to let users better engage in interacting with their own emotions. Our research also aims to explore whether an interactive system built around smart textiles could be used for emotion regulation.

\section{Related Work}

Emotion regulation and interactive textiles are two distinct domains, which both involve communication through the user's body and feelings. Advances in the development of smart textiles and emotion engaged interaction design have also helped drive the integration of these two domains.

\subsection{Emotion Engaged Interaction}

As we know, emotion not only has an impact on how we think and behave, but also plays a pivotal role in self-expression, social communication, and physical health. Norman [20] introduced the concept of emotional design, highlighted the importance of emotion in design, and took pleasant emotional experience as a standard to evaluate the success of a design product. In Picard's Affective Computing [24], she describes computing that relates to, arises from, or influences emotions. She tried to empower computer with the ability to recognize, understand, and even to have and express emotions, 
especially with wearable computers. With the advances in interaction design, affective design is no longer limited to the emotional data coding and presentation but turns to emotion engagement, affective loop interaction and experience centred soma design $[11,21]$, which emphasises the role of physical participation in the emotional interaction, including physiological signals participation and body movements participation.

In these studies, emotion-related bio signals such as electromyogram (EMG), blood volume pulse (BVP), electrodermal activity (EDA) and electroencephalogram (EEG) were extracted for biofeedback and embodied interaction. For example, Picard et al. [23] designed a pair of skin conductivity gloves to measure the wearer's skin conductivity levels as indicators of physiological arousal. This glove-like device used light to map the degree of arousal in the human body. The visual presentation can clearly show people's emotional arousal levels in different activities. The Brightbeat App [13] was designed for stress relief with users being able to observe their heartbeats; it slowed their breathing and improved self-reported calmness and focus. These design practices help users to understand their physiological state better, based on their subjective understanding and self-reflection.

Except for the emotion-related bio signal-based interaction, body motion was also explored within affective interaction research. These types of experiences were applied in Höök's SenToy Doll project [10], in which the user could express their physical and emotional state by using various kinds of emotional gestures to interact with a doll. In her another project, an instant messaging application could transform the user's gestures into emotional expressions - for example, when angry gestures were visualized into a strong aggressive colourful background [29], the receiver would then sense the emotion of the sender by interacting with the system.

Mood Swings is an interactive artwork designed by Bialoskorski et al. [2], which recognises affective movement characteristics expressed by a person and displays a colour that matches the expressed emotion. These design cases combine body biodata or movement with a feedback mechanism to achieve an affective experience and showed the potential of supporting emotional well-being. Users often experienced more autonomy in this process. On the other hand, the potential of smart textiles in this field has not been much developed, although they are more advantageous in supporting body and experience centred affective design.

\subsection{Affective Smart Textiles}

In affective smart textiles design, Stead [28] put forward her thinking about the relationship between fashion, emotion, and technology through practice-based research, and preliminarily investigated how clothing can both represent and stimulate emotional response through technology. Uğur [32] reported her study on the theory and design practice of wearing embodied emotions and designed several kinds of smart wearable products which expressed wearers' emotional state. She explored the definition of new body boundaries and the possibilities of expressing emotions in wearable design with technology and stated that emotional embodiment through wearable technology can help people to regulate their emotions. Ashford [1] more specifically researched the relationship between EEG signals and emotional states. In her projects, she attempted 
to integrate EEG signals into visualized embodied designs and found that potential users are very interested in the personalized aesthetics design of wearables and applied the design to inspire confidence with quantified self. In 2013, the SENSOREE company also designed a GER Mood Sweater collar to allow visualizing the user's emotional status with LED lights and brought it to the market [25]. The main function of these designs and research is to embody emotions on smart textiles and to arouse users' selfreflection and attention to them. Although these cases are textile-based augmented biofeedback and took advantage of e-textiles, they have not involved functional bidirectional biofeedback interaction between users and the textiles.

Another design case is the Affective sleeve [22], which produces rhythmic tactile movements with warm and slight arm pressure to promote calmness and reduce anxiety. The researcher found that the speed of the sleeve's tactile movements affects the participants' breathing rate and perception of calmness. It affects the user's emotional state with detailed vibrotactile feedback. According to the results, the feedback mechanism is also a potential factor to consider about in emotion regulation.

\section{$3 \quad$ Methodology}

The research followed a combination of the Research through Design (RtD) approach $[14,31]$ and mixed research method. Explorations during two design cycles served as stimuli to define the research direction and gather further feedback. In the first cycle, four types of modalities were integrated into textiles to represent biofeedback. These four modalities were evaluated during a qualitative user study, which resulted in the selection of visual, tactile feedback and hand gesture interaction as the starting point for the second design cycle. A shawl providing tangible emotional feedback was then designed. Touch motion was used for the user to interact with their embodied emotions. To verify the prototype's effectiveness and obtain further user feedback, a second mixed research evaluation was conducted with the prototype.

\subsection{First Design Cycle: Visualized Biofeedback on Textiles}

Table 1. Four feedback modalities for the smart textiles in our designs and their user feedback.

\begin{tabular}{|c|c|c|c|c|}
\hline Textile prototypes & Textile & $\begin{array}{l}\text { Feedback } \\
\text { modality }\end{array}$ & Description & User feedback \\
\hline & $\begin{array}{l}\text { Smocked } \\
\text { scuba knit- } \\
\text { ting fabric } \\
\text { with LED } \\
\text { lights }\end{array}$ & $\begin{array}{l}\text { Visual } \\
\text { feedback } \\
\text { (colour and } \\
\text { brightness) }\end{array}$ & $\begin{array}{l}\text { The LED light em- } \\
\text { bedded in 3D fabric } \\
\text { structure. The bright- } \\
\text { ness and colour } \\
\text { change according to } \\
\text { the emotional arousal } \\
\text { level. }\end{array}$ & $\begin{array}{l}\text { The LED light } \\
\text { mode is more in- } \\
\text { tuitive and effec- } \\
\text { tive. It has more } \\
\text { diversified forms } \\
\text { of expression. }\end{array}$ \\
\hline
\end{tabular}




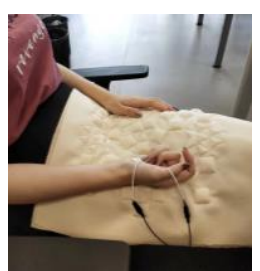

Quilted

scuba knit-

ting fabric

with vibra-

tion motors

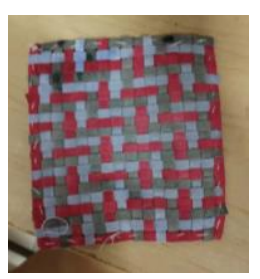

Overlapped non-woven

thermo-

chromic

stripes with

EeonTex

heater fabric

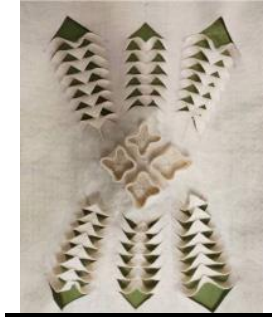

Laser-cut

non-woven

fabric with

motor
Tactile

feedback

(Vibration

frequency)

Thermal-

Visual

feedback

(red, blue,

black col-

our satura-

tion)

Shape-

changing

feedback

(Rotation

angle)
The vibration inten- Vibration mode sity of the motors in- is more private, side the textile the hand is more changes according to sensitive for vithe emotional arousal bration than the level. body.

Fabric is made of stripes, dyed with different thermochromic pigments. The temperature changes with the emotional arousal level, which leads to colour change.

The fabric texture surface moves driven by motors underneath with thread pulling the texture.
It has delayed emotional biofeedback, but easy to wear.

It feels cool, has a better visual effect, suits the younger user, but soft material is not easy to manipulate.

Emotion expression and recognition is the premise of emotion regulation. So, at the beginning of the first design cycle, it was our goal to explore the design space of textilebased biofeedback visualizations. Following the RtD approach, we first designed several textile-based biofeedback visualizations and observed how the user would interact with these smart textile prototypes to drive the design and further research inquiries.

Four kinds of textile prototypes were designed that could represent the user's emotional arousal level based on different feedback modalities: light, vibration, colour change (based on thermochromic ink), and shape change. Table 1 shows an overview of the four textile prototypes. An EDA sensor placed on the finger measured the user's emotional arousal level. The data was then used as input for the visualization in the textiles.

During the evaluation, six participants were invited to interact with these textiles and place these textiles in their comfortable position, whether attached or spread around parts of their body. Most participants preferred to place the fabric on their upper body (near their arms and chest) where they can touch it, while their emotional arousal level was displayed using the feedback modality.

After the participants finished interacting with the prototypes, they were interviewed about their subjective experiences. Two participants stated that they would not mind if other people see their emotional states, while four participants were very concerned about their privacy. Most participants mentioned that the visualization of emotions 
could be more useful for overly stressed people, while healthy (less stressed) people do not necessarily need to visualize their emotions all the time. It could be important for people under chronic, continuous stress to have ways to vent (or let out) their negative emotions to prevent them from bottling up these feelings.

Some key reactions from participants about the different types of feedback modalities are summarized in the last row of Table 1. Overall, visual and tactile feedback modalities are more widely accepted, because they can be easily perceived and placed around users' body and have more diversified forms of expression. They were concerned about the thermochromic feedback, because the temperature change was too slow to feedback the EDA level. Some participants showed interest in the fabric shape changing caused by the mechanical motor-for example, they said it feels interesting to have the fabric move on their body. Also, they thought young people may be more likely to accept the shape changing fabric, but the fabric structure of the sample is relatively soft and unstable. When considering the possibility of daily use, they agreed that visual feedback and vibration feedback were more realistic. Besides, participants said that they did not want to wear them every day as the form of clothes, but they would like to wear them as accessories like a scarf or wristband which are more flexible.

In the interaction between participants and smart fabrics, we observed that they showed more interest in some fabrics with special texture and would like to keep touching them. The initial fabric texture design was to better integrate textiles and electronic components, such as the smoked sewing texture in visual feedback and the quilted sewing structure in vibration feedback. During the study, participants used their hands and fingers to poke the bubble-like fabric textures. When asked about this behaviour, participants mentioned that it reminded them of the action of popping bubble in certain plastic wraps. This action could help them to release their stress because the action of pressing the material combined with the sound of the popping bubbles made them feel more relaxed. However, they did not take many actions on the thermochromic and motion feedback textiles. Except for the reflection caused by being able to visualize their emotions, this insight could help frame the design of interactions that deal with motions $[17,18]$, and led us to ask whether we can regulate the user's emotion through motionbased interaction and how-to bring motion-based interaction into the smart fabric system.

\subsection{Second Design Cycle: Designing the Interactive Smart Shawl}

According to the first experimental results and user feedback, a new prototype was then designed which could be used to further explore the role of interaction for self-regulation. The final prototype is a shawl, designed for people who want to regulate their emotions in real, actual environments during their daily activities, such as going for outdoor activities, communicating with people, etc. This design is expected to help users balance their emotions and reduce the negative impact of aggressive emotions on their health and social life (see Fig. 1). 

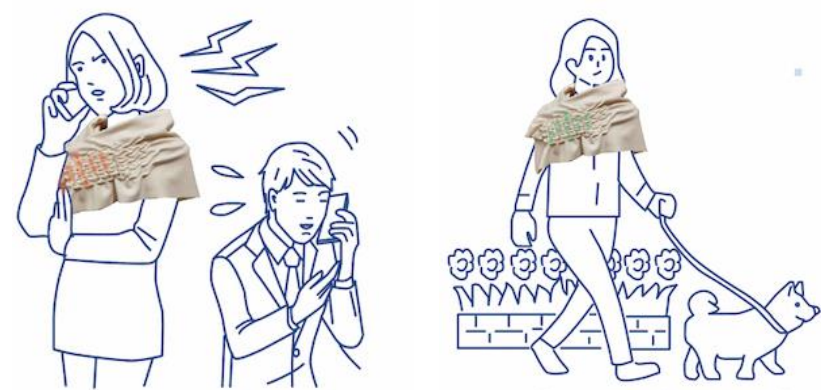

Fig. 1. User scenarios: The smart shawl aims to help people to self-regulate emotions in their daily lives, like social activities or outdoor activities.

Emotions are complicated and complex, there is no single bio-signal that can accurately capture all the emotional states. Some studies have shown that a combination of multiple biological signals can be used to determine users' emotion $[12,15]$. Because the focus of this research is on the exploration of how movement-based interaction plays a role in the self-regulation of emotion, we have not focused on emotion recognition technology. Therefore, we selected a Grove EDA sensor to measure the general emotional arousal level.

Based on the reactions from the participants during the exploratory study, the light feedback and vibration feedback were more preferred for visualizing and sensing the dynamic data from the EDA sensor. Moreover, an interactive action was added to the visualization that could be utilized when users feel stressed or emotionally aroused. Inspired by the action of popping bubble wrap, users can relieve their stress by poking each fabric bubble to change the emotion arousal triggered from the light or vibration feedback, thus allowing them to calm their emotion down (see Fig. 2). When the user feels nervous, the location of the shoulder and chest is more natural to provide comfort; therefore, the product is designed as a shawl, which users can wear on their shoulders.

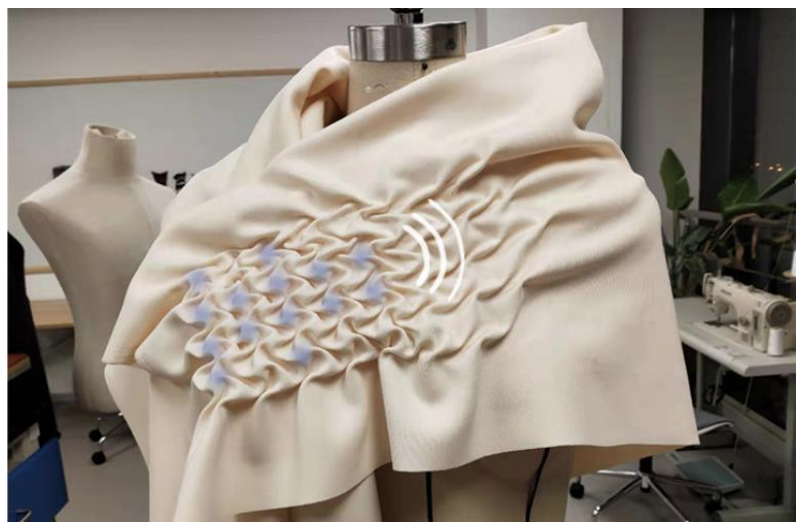

Fig. 2. User scenarios: The smart shawl aims to help people to self-regulate emotions in their daily lives, like social activities or outdoor activities. 


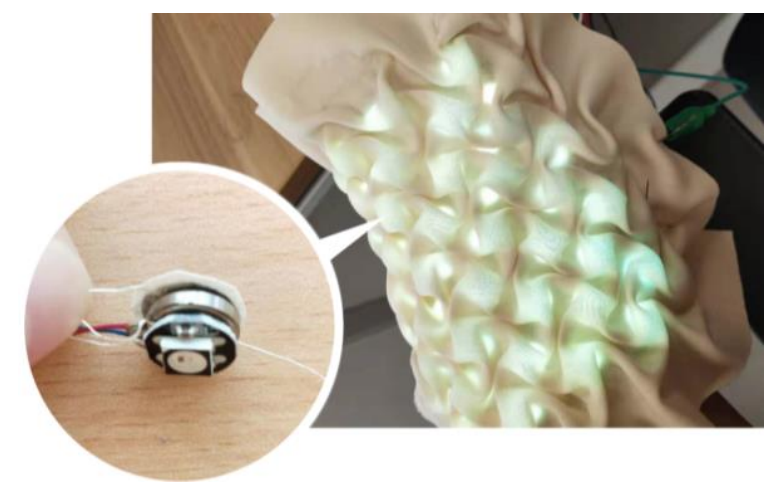

Fig. 3. The electronic components integrated with the pressure sensor, LED and vibration module, as an independent interaction unit.

Smart Shawl Prototype Composition. The functional part of the shawl consists of 14 separate fabric bubbles, each of which integrates a pressure sensor made by Velostat foil (pressure-sensitive material based on piezo-resistive effects), a LED light, and a vibration motor. When the wearer's arousal level changes, each fabric bubble module will react by changes in intensity (see Fig. 3).

Interaction Modes and Interaction Flow. The smart shawl has two modes for users to choose in different scenarios, the light and vibration modes. The light mode is a public mode, in which the people around the wearer can perceive the wearer's emotional state through the colour and brightness of the light. In the weak emotion arousal state, it will light up slightly with tint colour. In a stronger emotion arousal state, the light will be brighter and show stronger colour changes. The vibration mode is considered as the privacy mode because its feedback can only be sensed by the wearers themselves. Vibration intensity also changes depending on the emotional arousal level as measured by the EDA sensor.

The interaction flow is displayed in Fig. 4. The shawl first visualizes the wearer's emotional state through light or vibration, which allows the wearer to be aware of and reflect on their emotional state; based on this visualization they can further interact with the smart shawl. In the light mode, when the wearer feels stressed or nervous, the EDA sensor measures the state after which, the light would turn on, and the wearer could poke each fabric bubble with his/her finger for a brief period to make the corresponding light to turn off. The vibration mode works this way as well. The module stops working as feedback to the user's behaviour. The wearer can repeat this interaction with the smart shawl constantly to relieve their stress or let out their negative emotions until they are somewhat calmer. Based on the prototype the shawl was tested to verify whether it could help wearers to self-regulate their emotion. 


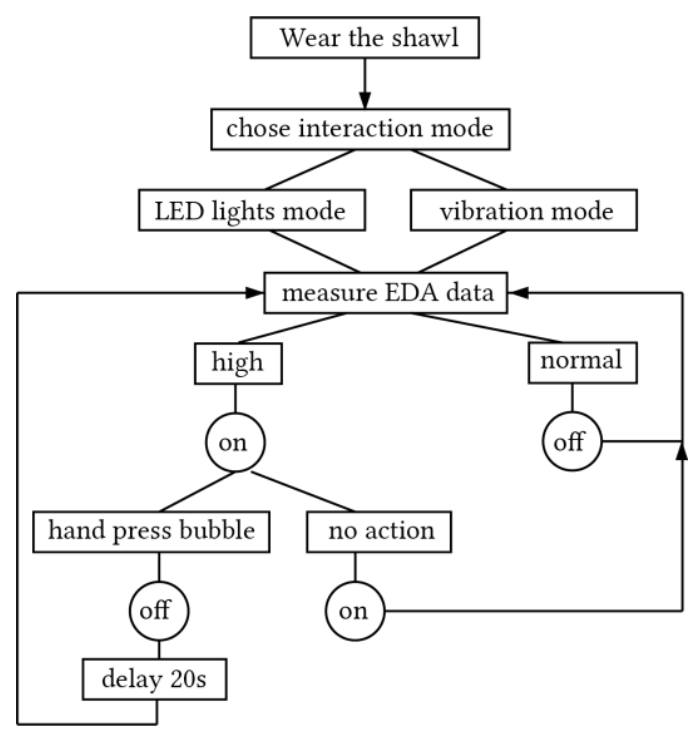

Fig. 4. The interaction flow of each textile bubble.

The Emotion Regulation Loop. In this study, emotion regulation was implemented based on the following three steps. In the first step, the emotion data was acquired by the EDA sensor and processed to identify the emotion variation level based on the wearer's emotional arousal. In the second step, the emotional state was visualized, which would transform the arousal data into lights or vibration within the smart textiles. In the third step, the user could interact with the smart textiles to self-regulate their emotions. The shawl would show the state of the user's emotional arousal and allow the user to interact with the visualized emotions. Moreover, because of the interplay between the emotions and the user's actions, their emotions could change continuously. As such, this process could form a dynamic loop to guide the user back to the ideal emotional level. Besides, interaction can be both conscious and unconscious. If it is a conscious interaction, there could be a reflection step before interaction and the user's reflection may also affect his or her emotion (see Fig. 5). 


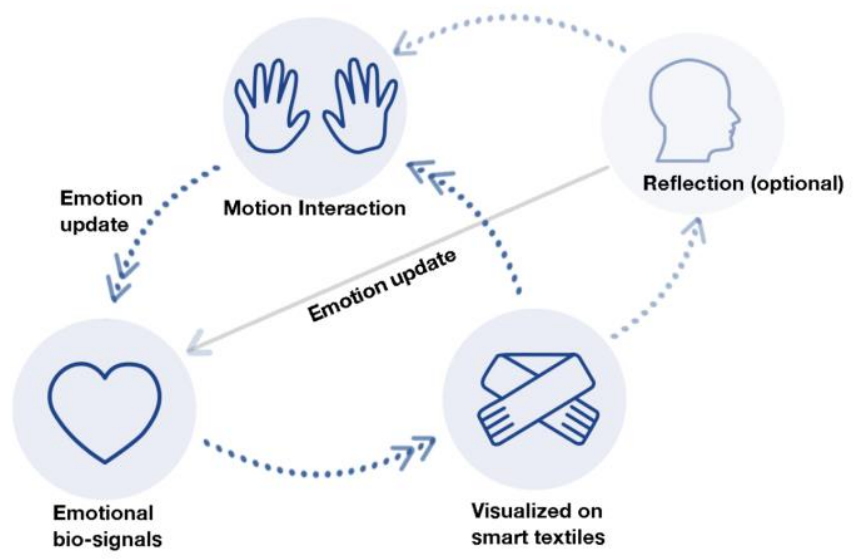

Fig. 5. Emotion regulation loop process with visualized biodata in the smart shawl prototype.

\section{Smart Shawl Prototype Experiment}

We recruited 15 unpaid volunteers from a local University to participate in the experiment. There were 9 females and 6 males (aged between 18 and 26; Mean $=21.2, \mathrm{SD}=$ 2.072). They came from different backgrounds, including industrial design, architecture, computer science, media art, and were all recruited from a social media platform. The participants were selected based on their interest to participate in this study. The experiment was carried out in a design studio. To explore the attitude of the users, an emotion induction interview and a semi-structured interview were conducted. During the interviews, we recorded participants' EDA data for quantitative analysis and their reactions to the prototype for qualitative analysis. The test had the following three goals:

- To further understand the participants' attitude towards emotion regulation and how they handle it;

- To induce the emotional response of the participants, then test the effect of the prototype for emotion regulation;

- To elicit participants' experience and feedback on the prototype.

\subsection{Procedure and Apparatus}

According to the objective of the study, a preliminary semi-structured interview outline was compiled, and four students were pre-interviewed based on the outline. Two emotion induction methods were compared: video emotion induction and recall emotion induction [27]. Based on the results and feedback from the participants, the recall emotion induction method was selected since it resulted in a stronger emotional response from the participants (measured by the EDA sensor), while the video induction method resulted in too much variance between the participants based on their personal prefer- 
ence for video content - that is, for the same video content, some people are more excited, some people are more insensitive. The outline of the interview was finally formed after the modifications.

Before the interview began, the general interview process was introduced, including duration, purpose, and the prototype itself. The participants signed informed consent to voluntarily participate in the study (see Fig. 6). Then participants were interviewed with the following pre-set questions.

- Are you bothered by emotion problems in your daily lives?

- What kind of emotion are you dealing with? And which scenario?

- How do you deal with this kind of emotional fluctuation in their daily life?

- What's your explanation of your coping behaviour?

After the interview, several questions were asked for emotion induction.

- When did your last emotional fluctuation happened?

- Could you recall what happened?

Then participants filled in the first Self-Assessment Manikin (SAM) form [3]. After the emotion induction, the participant was encouraged to experience the prototype. Finally, the participants were asked to self-evaluate their emotions again with the second SAM form and express their feelings and opinions about the prototype.

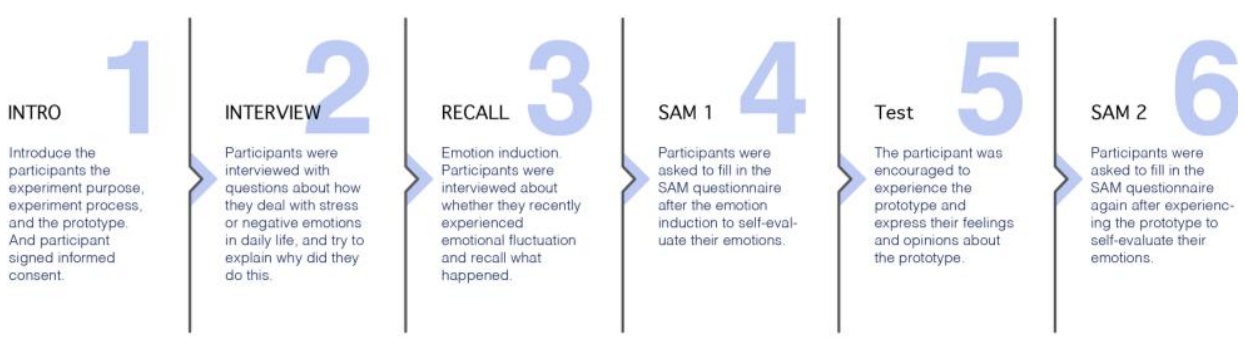

Fig. 6. Steps of the experiment process.

\subsection{Results}

The data collected during the interview included data from the EDA sensor, video recordings and written text on the forms. This data was used as input for the analysis to evaluate the prototype. Within the data presented in this section, $\mathrm{F}$ and $\mathrm{M}$ represent the gender of the participants, and the numbers after the letters represent the order of the participants.

Coping Behaviours of Emotional Fluctuation. As part of the unstructured interview, we asked the participants to recall their behaviour after they experienced stress or strong emotions in their daily lives and to give their interpretation for these behaviours. Their coping behaviours vary, including tearing paper, doing exercises, hugging people, etc. 
Based on their responses, we were able to categorize them into five types of interpretation: positive intervention, venting negative emotion actions, increasing sense of control, attention diversion and unconscious behaviours they could not explain clearly. The full list of behaviours and our interpretations grouped in categories are summarized in Table 2.

Table 2. The participants' behaviours and their interpretation when dealing with stress or emotion variations.

\begin{tabular}{ll}
\hline Interpretation of their actions & $\begin{array}{l}\text { Behaviours when participants were experiencing } \\
\text { stress/emotion variation }\end{array}$ \\
\hline $\begin{array}{l}\text { Positive intervention } \\
\text { Venting actions }\end{array}$ & $\begin{array}{l}\text { Hugging other people or plush toys. } \\
\text { Paper tearing, tearing other things apart, rubbing hands, } \\
\text { doing exercise, running, poking bubbles, throwing the } \\
\text { pillow. }\end{array}$ \\
Increase control & $\begin{array}{l}\text { Pressing a pen. } \\
\text { Pulling hair, playing phones. } \\
\text { Attention diversion }\end{array}$ \\
Unconsciousness behaviour & $\begin{array}{l}\text { Walking up and down, scratching head, swinging hands, } \\
\text { swinging feet, more gestures. }\end{array}$ \\
\hline
\end{tabular}

Emotion Induction and EDA Data. The emotion induction method evoked fluctuation in participants' emotions. Fig. 7 shows each participants' average EDA data before and after emotion induction. A paired t-test on the data showed there was significant difference $(t=10.512, P<0.05)$. Specific comparisons showed that the average EDA value after emotion induction $(218.33 \pm 54.86)$ was significantly higher than the average EDA value before induction $(71.67 \pm 21.85)$.

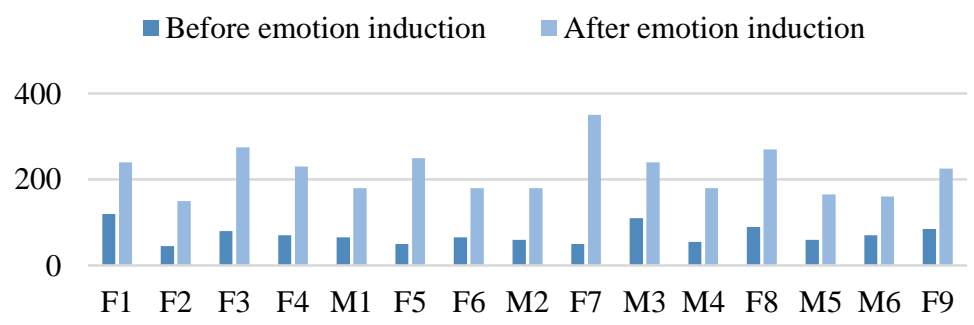

Fig. 7. The participant's EDA data comparison before and after emotion induction.

SAM Tests Before and After Experiencing the Shawl. Based on the assumption that participants' emotional reflection is significant, the SAM test was conducted to evaluate the participants' subjective emotional variations. We invited the participants before and after interacting with the prototype to complete the SAM questionnaire, which measures emotional variations on a scale from 0-9 for three aspects: emotional valence, 
arousal, and dominance. Fig. 8 shows the graphs of the values for the three SAM aspects. The values of valence 1 , arousal1, and dominance 1 are the data after the emotion induction interview, but before they interacted with the prototype. Emotional valence2, arousal2, and dominance 2 are the data after they interacted with the prototype.

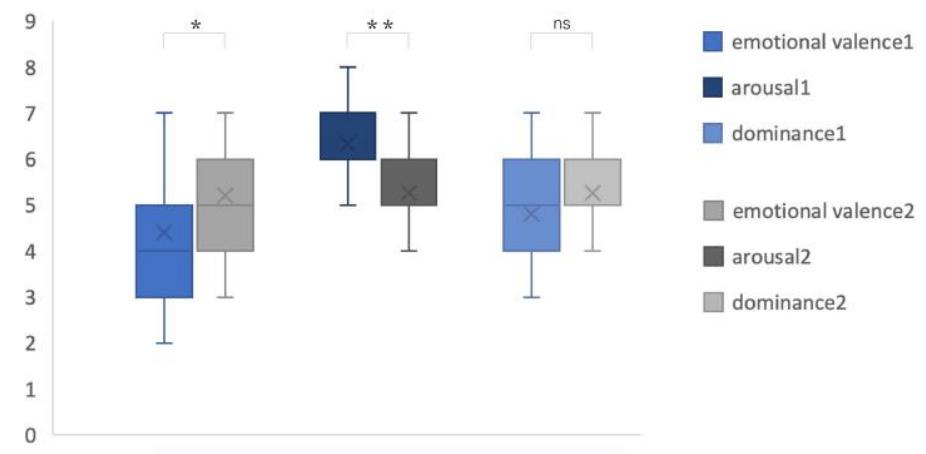

Fig. 8. The participants' SAM box $\operatorname{plot}\left({ }^{*} \mathrm{P}<0.05,{ }^{* *} \mathrm{P}<0.01,{ }^{* * *} \mathrm{P}<0.001\right)$.

We did 3 paired t-tests on the data. The results are as follows: (1) The emotional valence value increased from $4.40 \pm 1.35$ to $5.20 \pm 1.08$, after the completion of the test, and it was found that the difference between the two groups was significant, $\mathrm{t}=-2.567, \mathrm{P}=$ $0.022<0.05$, indicating that the interactions with the prototype improved the general positive emotion of the participants. (2) After the experiment, the results of emotion arousal level showed that the difference between the two conditions was significant, $t$ $=5.870, \mathrm{P}<0.01$. It shows that the prototype is conducive to the recovery of users' emotion arousal level. (3) Although the emotion dominance score increased from 4.80 \pm 1.15 to $5.27 \pm 0.88$, there was no significant difference between the two conditions after the experiment.

User Feedback. Participants were encouraged to express their feeling and opinions when they were interacting with the shawl prototype. They gave their feedback about the shawl, the relation between the shawl and their body (for example, where else could the textiles be worn), and whether the poking bubble interaction could help them regulate their emotions. F1 mentioned that the LED light could be a disturbance for her because every time the lights turned on, she felt the need to reflect on herself. M1 and F3 discussed that the LED lights could help to distract their attention when being stressed. M1, F5, F6 remarked that the bubble textiles could have more sources of multisensory feedback, such as a click sound and vibration. M5 suggested that the LED lights could light up one by one, while simultaneously creating rhythmical musical feedback.

Regarding the form of poking bubble in the textile, some participants liked the shawl and would like the functional part to be placed around the shoulder as they thought it could be more comfortable. F5 suggested that the smart shawl could also integrate a hug function and heating function to give comfort to the user. F8 preferred it to be placed on the arm or hand, so it could be easier to wear, and its reaction could be seen more clearly. The participants also had some concerns, because the shawl might not be 
tight enough to be in contact with the skin, and the user had to look down at the fabric to see the lights when it was in LED light mode. F4 mentioned that the interactive textile could also be used in handbags for women, for example by measuring the EDA data when they were holding the bag. F7 suggested it should be integrated into a wristband. M5 thought it could be placed around the user's waist. This participant recalled that when he is angry, he would put his hands on his waist. Most of the participants preferred to keep both the public LED light mode and the private vibration mode so they could be able to switch between the modes according to the scenario. The light mode is more visual and vibration mode also has the massage function.

F7 thought that the interaction with the bubbles was not enough for her to let her emotions out because she prefers more energy-consuming exercises. M2 mentioned that for real users, the smart textile form and interaction mode should be customized based on personal preferences, and users might want their relatives involved in the interaction. F1 and M4 thought that the prototype did not affect them. Most of the participants gave positive feedback after they experienced the interaction and thought it could help people with emotional self-regulation. Some of them mentioned their expectations for smart textile products with improved performance.

\section{Discussion}

\subsection{Two Design Cycles}

Following the RtD approach, four smart textiles and one smart shawl were designed to help us explore the interactive smart textile design for emotion regulation and verify its feasibility with the hybrid research method.

In the first design cycle, we tested four smart textiles and interview and got the user's feedback on emotional visualization and feedback mode based on different types of smart textiles, then chose visual and vibrotactile as the feedback modes. Another discovery is the gesture interaction triggered by fabric samples in the RtD process. We realized that both body motion and interaction have an impact on emotional state. Therefore, the second study attempts to combine movement and feedback mechanism with interaction to amplify the effect on emotion.

The results of the second design cycle indicated that the movement-based interactive emotion self-regulation design of the smart shawl could help the stressed user to reflect and manipulate their emotional states.

From the participants' feedback, it seems that each person's emotions might be aroused by different triggers, but the negative emotional experiences are similar, such as depression, impatience, nervous, etc. In Table 2, we tried to categorize participants' instinctive responses to emotions into four types. The participants' interpretations of these behaviours are not necessarily the only ones, since there are many complex factors behind emotion and response behaviours. However, the participants' explanations may help us to design effective interactions strategies for emotion regulation.

After the interview emotion induction, the SAM questionnaire results show that, in general, the prototype had a greater impact on the participants' emotional valence and arousal after they interacted with the prototype. From the qualitative interviews, we 
found that most participants showed a positive attitude towards the design of the prototype and put forward their suggestions for further improvements. Male participants preferred to position the smart textiles on the arm or hand, while female participants wanted to place it around the chest. The participants' response is consistent with Uğur's survey [32] on the somatic sensation of emotions, which indicates that the chest and shoulders have more feelings when participants are emotionally aroused. Some participants also expressed the expectation that the interactive textile prototype could be combined with gloves, wristbands, and handbags, to further integrate it into their daily life. Many participants expressed their expectations for richer interactive feedback. For example, when visualizing emotion with lights or vibration, there could be additional sound feedback or interaction guidance to achieve a better interactive experience and emotional relief.

\section{$5.2 \quad$ Reflection}

Several aspects may affect the emotional status of participants in the experiment, such as the speed of emotional recovery of the participants, the novelty of a new prototype, personal preferences of materials, etc. Above all things, movement-based interaction and feedback mechanism are the most important factors.

In previous studies, it has been suggested that there is a bi-directional relationship between emotion and body motor behaviour [5,6]. Body movements can be used not only to express emotions but also to influence how we feel due to the mirror neurons. Body movement exercises, such as TaiChi, yoga [8], have long been used in emotion regulation and this concept has been applied in dance/movement therapy. However, what remains unclear is how to design motion-based interaction to affect emotion. Hand Interactions such as popping sealed air capsules have been shown to be effective for nervous or stressed individuals since it can help to release muscle inhibition [7]. These types of interactions have also been applied in digital user interfaces. For example, the NetEase music application recently introduced a stress release mode in which users can poke virtual bubbles or break the virtual glass on the screen to relieve stress [19] and received positive feedback from users.

Emotion self-regulation methods through body motion can be determined by different types of emotions. Besides poking fabric bubbles, we may consider different types of interactive hand actions, and a wider range of body actions for interaction. Compared with the physiological state, we can better control our body movement, muscle movement and respiratory rhythm to a certain extent. This advantage should be more integrated into the interaction design for emotion regulation, rather than just receiving the information conveyed by biofeedback.

In the smart shawl, the feedback mechanism plays multiple roles: (1) Visualizing the emotion-related physiological information; (2) Causing users to reflect on their emotional state; and (3) Motivating users to interact with their emotion visualizations with actions. Different feedback modes also affect users' emotional state. In our design, we let users choose their own preferred feedback modalities to interact with the smart shawl. Although the results of SAM test show that the overall both modalities results seem to be effective on emotional regulation, the difference of impact between two 
feedback mechanisms remains unknown, which is the part we will follow up in the future research.

Future developments of smart textile products do not only concern technological breakthroughs but also about designing interaction styles that fit these close-to-thebody products to improve the user's experience. There is an intimate relationship between the human body and the products we carry around, such as our clothing. Smart textile products, in particular, will be more dynamic and flexible and can be tailored according to the user's physical and psychological states. Embodied interaction design can further expand the functions of smart textiles while improving users' emotional experience. This can help them to further communicate with themselves and others, based on their emotions in a dynamic and real-time basis.

\subsection{Further Development}

To test the effect of the movement-based interaction in smart textiles, the prototype design is based on a simple interaction model. In the future, we plan to design richer interactions in the system to improve the user experience. Also, we will explore further usage scenarios and user acceptance and feedback in real-life contexts.

Further, when designing interactions based on emotions it is necessary to consider the appropriate interaction location on the body, fabric selection, emotion visualization modes, and other kinds of physiological signals which can be used to measure emotion states. How to personalize the emotional self-regulation products according to the different scenarios to achieve the best user experience will be a topic for further research.

\section{Conclusion}

The affective interaction of the prototype is self-supportive, a closed-loop, avoids privacy issues, gives users autonomy and, at the same time, helps users to understand and interact with their embodied emotions. Because many factors affect human emotions, it is difficult to design interactions which react to a specific aspect of our emotions. However, the emotional experience can be designed. In this case, user's emotional experience was affected through embodied interaction. Our prototype helps people to regulate emotion by letting them see and 'interact' with them. The emotion regulation smart textile system may also benefit the family members or friends since they are also closely related to users' emotional states. The research application can be extended to the design of emotion regulation for social relationships. According to users' cultural backgrounds and preferences, products can be designed for self-supportive users and also for users to communicate with their families or friends.

The approach of this research is based on Research through Design. The process of combining making and testing in iterative cycles helped identify the research direction and reflect on the research question. With the development of interactive textiles, designers will face the situation of solving multi-disciplinary involved design problems. The proposed smart textile design methods and experiences will help them explore functional and aesthetic possibilities in their projects. 


\section{Acknowledgement}

The authors would like to thank all the participants for the time and the reviewers for their comments. This research was supported in part by Xi'an Jiaotong-Liverpool University (XJTLU) Key Program Special Fund (KSF-A-03), and XJTLU Research Development Fund (RDF-17-01-54).

\section{References}

1. Ashford, R. Responsive and Emotive Wearable Technology: physiological data, devices and communication, Doctoral dissertation, Goldsmiths, University of London. (2018).

2. Bialoskorski, L. S., Westerink, J. H., \& Van den Broek, E. L. Experiencing affective interactive art. International Journal of Arts and Technology, 3(4), 341-356. (2010).

3. Bradley, M. M., \& Lang, P. J. Measuring emotion: the self-assessment manikin and the semantic differential. Journal of behavior therapy and experimental psychiatry, 25(1), 49-59. (1994).

4. Cherenack, K., \& van Pieterson, L. Smart textiles: Challenges and opportunities. Journal of Applied Physics, 112(9), 091301. (2012).

5. Damasio, A. R. The feeling of what happens: Body and emotion in the making of consciousness. Houghton Mifflin Harcourt. (1999).

6. de Rooij, A. Toward emotion regulation via physical interaction. In Proceedings of the companion publication of the 19th international conference on Intelligent User Interfaces, pp. 57-60. (2014).

7. Dillon, K. M. Popping sealed air-capsules to reduce stress. Psychological reports, 71(1), 243-246. (1992).

8. Francis, A. L., \& Beemer, R. C. How does yoga reduce stress? Embodied cognition and emotion highlight the influence of the musculoskeletal system. Complementary therapies in medicine. (2019).

9. Fritsch, J. Understanding affective engagement as a resource in interaction design. Nordes 3. (2009)

10. Höök, K., \& Laaksolahti, J. Empowerment: a strategy to dealing with human values in affective interactive systems. (2008).

11. Höök, K. Affective loop experiences-what are they? In International Conference on Persuasive Technology, pp. 1-12. Springer, Berlin, Heidelberg. (2008).

12. Jeon, M. Emotions and affect in human factors and human-computer interaction: Taxonomy, theories, approaches, and methods. In Emotions and Affect in Human Factors and $\mathrm{Hu}-$ man-Computer Interaction, pp. 3-26. Academic Press. (2017).

13. Khut, G. Designing Biofeedback Artworks for Relaxation. In Proceedings of the $2016 \mathrm{CHI}$ Conference Extended Abstracts on Human Factors in Computing Systems, pp. 3859-3862. (2016).

14. Koskinen, I., Zimmerman, J., Binder, T., Redstrom, J., \& Wensveen, S. Design research through practice: From the lab, field, and showroom. Elsevier. (2011).

15. Lisetti, C. L., \& Nasoz, F. Using noninvasive wearable computers to recognize human emotions from physiological signals. EURASIP Journal on Advances in Signal Processing, 2004(11), 929414. (2004).

16. Mota, S., \& Picard, R. W. Automated posture analysis for detecting learner's interest level. In 2003 Conference on Computer Vision and Pattern Recognition Workshop, Vol. 5, pp. 4949. IEEE. (2003). 
17. Nanjappan, V., Shi, R., Liang, H. N., Lau, K. K. T., Yue, Y., \& Atkinson, K. Towards a Taxonomy for In-Vehicle Interactions Using Wearable Smart Textiles: Insights from a UserElicitation Study. Multimodal Technologies and Interaction, 3(2), 33. (2019).

18. Nanjappan, V., Shi, R., Liang, H. N., Xiao, H., Lau, K. K. T., \& Hasan, K. Design of Interactions for Handheld Augmented Reality Devices Using Wearable Smart Textiles: Findings from a User Elicitation Study. Applied Sciences, 9(15), 3177. (2019).

19. Netease Cloud Sati Space Introduction. https://baijiahao.baidu.com/s?id=1620172495110902700\&wfr=spider\&for=pc. Last accessed 2020/6/10

20. Norman, D. A. Emotional design: Why we love (or hate) everyday things. Basic Civitas Books. (2004).

21. Nunez-Pacheco, C., \& Loke, L. Crafting the body-tool: a body-centred perspective on wearable technology. In Proceedings of the 2014 conference on Designing interactive systems, pp. 553-566. (2014).

22. Papadopoulou, A., Berry, J., Knight, T., \& Picard, R. Affective Sleeve: Wearable Materials with Haptic Action for Promoting Calmness. In International Conference on Human-Computer Interaction, pp. 304-319. Springer, Cham. (2019).

23. Picard, R. W., \& Scheirer, J. The galvactivator: A glove that senses and communicates skin conductivity. In Proceedings 9th Int. Conf. on HCI. (2001).

24. Picard, R. W. Affective computing. MIT press. (2000).

25. Sensoree. Mood Sweater collar to visualize the user's emotion. http://sensoree.com/, Last accessed 2020/6/10

26. Shafir, T. Movement-based strategies for emotion regulation. Handbook on emotion regulation: Processes, cognitive effects and social consequences, 231-249. (2015).

27. Siedlecka, E., \& Denson, T. F. Experimental methods for inducing basic emotions: A qualitative review. Emotion Review, 11(1), 87-97. (2019).

28. Stead, L. J. 'The emotional wardrobe': a fashion perspective on the integration of technology and clothing, Doctoral dissertation, University of the Arts London. (2005).

29. Sundström, P., Ståhl, A., \& Höök, K. eMoto: affectively involving both body and mind. In CHI'05 extended abstracts on Human factors in computing systems, pp. 2005-2008. (2005).

30. Terry, M. L., \& Leary, M. R. Self-compassion, self-regulation, and health. Self and Identity, 10(3), 352-362. (2011).

31. Toeters, M., ten Bhömer, M., Bottenberg, E., Tomico, O., \& Brinks, G. Research through design: a way to drive innovative solutions in the field of smart textiles. In Advances in Science and Technology, Vol. 80, pp. 112-117. Trans Tech Publications Ltd. (2013).

32. Uğur, S. Wearing embodied emotions: A practice based design research on wearable technology. Milan, Italy: Springer. (2013).

33. Wensveen, S. A., Djajadiningrat, J. P., \& Overbeeke, C. J. Interaction frogger: a design framework to couple action and function through feedback and feedforward. In Proceedings of the 5th conference on Designing interactive systems: processes, practices, methods, and techniques, pp. 177-184. (2004).

34. Yu, B. Designing biofeedback for managing stress. (2018).

35. Yucha, C., \& Montgomery, D. Evidence-based practice in biofeedback and neurofeedback. Wheat Ridge, CO: AAPB. (2008). 